RESEARCH REPORT

\title{
ANALYSIS OF SENSORY PROCESSING ABILITIES AMONG AUTISM SPECTRUM DISORDERS CHILDREN
}

\begin{abstract}
\section{BACKGROUND AND AIMS}

Autism is characterized by the complex paradigm of intellectual disabilities that leads to impairment in sensory integration, communication barriers and social interaction. Therefore, the purpose of this study is to analyze the sensory processing abilities among autism children. Further the study will be helpful in identifying difficulties faced by autistic children in multidimensional aspects.
\end{abstract}

\section{METHODOLOGY}

A cross-sectional study was conducted at special education institute/schools of Karachi among the Autism Spectrum Children to evaluate the patterns of sensory integration by using Short Sensory Profile.

\section{RESULTS}

A total of 50 children, 23 males and 27 females with mean age of $9.5 \pm 1.84$ years included in the study. On SSP, the mean scores of participants were 17.89 \pm 7.61 in tactile sensitivity, $16.66 \pm 6.90$ in Under-responsive/Seeks Sensation, 14.45 \pm 5.75 in Visual/Auditory Sensitivity, $17.77 \pm 7.04$ in Auditory Filtering, 19.57 \pm 7.91 in Low Energy/Weak while lowest scores were reported in Taste/Smell Sensitivity and Movement Sensitivity.

\section{CONCLUSION}

It was concluded that definite difference is more prevalent among children with autism whereas varying percentages are found on different items on Short sensory profile. Further studies are required to assess the sensory processing issues for more useful outcomes.

\section{KEYWORDS}

Sensory Integration, Autism, Behavior, Social, Cognition, Developmental Disabilities, Hypersensitivity

\author{
Sundas Khan \\ Physiotherapist \\ Hayat-e-Nau Rehabilitation Center for \\ Disable Children \\ sundaskhan_2013@hotmail.com
}

[Khan S. Analysis of Sensory Processing Abilities among Autism Spectrum Disorders Children. Pak. j. rehabil.2019;8(2):38-43] 


\section{INTRODUCTION}

Autism is characterized by the complex paradigm of intellectual disabilities that leads to impairment in sensory integration, communication barriers and social interaction'. Centre for disease control and prevention formed the Autism and Developmental Disabilities Monitoring (ADDM) System to gather facts and figures which provide estimates of the prevalence of Autism Spectrum Disorder in the United States But tracking and determining the prevalence of ASD give rise to distinctive challenges like lack of biologic diagnostic markers, heterogeneity day to day changing in diagnostic criteria ${ }^{2}$. ADDM provide data on ASD prevalence among children aged 8 years that shows increase number from approximately one in 150 children in 2002 to one in 68 in 2012 in multiple United State communities, more than doubling in this period ${ }^{3}$. However, there is no cure for autism as some of the studies showed that regular treatments may be effective to make the child independent with improved quality of life but not meaningful to control the disability ${ }^{4}$. Moreover, therapies like music, speech and physiotherapy leads to the betterment of the child in order to enhance interaction and social well-being ${ }^{5}$. However, there are domains that are severely being affected in autism, particularly sensory integration process is found to be impaired among children with intellectual disabilities ${ }^{6}$.

Sensory system evaluation is important for the diagnosis of autism. It was highlighted in one of the study that autistic children develops hypersensitivity in response to any stimuli due to sensory processing overload that ultimately affect their social interaction and disturbs the normal functions thereby leads to sensory dysfunction?. These aspects lead to adverse consequences of intellectual disability. An empirical investigation based on sensory dysfunction and impairments in autistic children was conducted using a sensory profile. It was determined that marked frequent responses to sound were observed in autism associated dysfunctions whereas no response has been observed in first two years of autistic children ${ }^{8}$. Moreover, sensory profile was helpful to discriminate the autism based on the ranking of 8-10 that has been significantly scored among autistic individuals. In contrast, it was reported that autism children possessed higher rate and great differences in sensory-perceptual abnormalities in comparison to Down syndrome and normal children?.

A retrospective study reported that approximately 200 children are annually diagnosed with autism with severe pattern development ${ }^{10}$. Moreover, Weider and Greens described that most of the autistic participants experienced difficulties in the auditory response or problems while some children may suffer from auditory hypersensitivity". Another study observed that auditory stimuli is a strong tool that helps to distinguish between children with or without autism as it is substantial to assess sensitivity in infancy ${ }^{12}$. Further- more, another study described that there is a disturbance in the pattern of sensory modulation and processing thus affecting the whole sensory process or systems. It was found that $80 \%$ children aged less than 6 years diagnosed with autism had findings that $51 \%$ had no response to sound, $81 \%$ had sensitivity to loud noises, $53 \%$ showed visual inspection on hands or fingers, whereas $62 \%$ children showed arm flapping ${ }^{13}$.

Previous studies reported that some of the parents might face difficulties with their children that indicated the first sign of autism. Furthermore, it was observed that infants with autism do not response to certain sounds and sensitive to taste and pain as well. However, a difference was observed among infants in sensory processing that either they were sensitive to touch, showed no interest or over excitement, showed no interest in communication and like or dislikes the moving objects. These responses helped the parents to identify the difference between children with autism sensory disorder ${ }^{14}$. Furthermore, the children who were commonly developing mannerisms, lack of sensations, interest and inability of identifying objects were preceded for the diagnostic interview of autism. Moreover, studies concluded that these behaviors with respect to social, sensory and communication aspects are helpful to categorize the difference between autistic and children with developmental delay ${ }^{15}$.

According to Hobnon, the responses in autism are dependent upon the quality of sensory stimuli either from the environment, events or individuals. Therefore effective sensory integration therapy is recommended in autism as number of studies demonstrated that interventions that enhances the sensory integration are useful for the autistic children to improved their sensory processing, so that they could easily interact with the environment thereby learn effectively to accomplish functional tasks. On the contrary, most of the individuals of autism exhibit complex sensory behavior disorders that included inappropriate skills performance, poor socialization, loud screaming or shouting, frequent urination or harming their body part by biting their own body skin ${ }^{16}$.

Therefore early interventions are indicated for sensory disorders in autism. As sensory processing is crucial to help in reasoning and play important role in concentration or cognitive functioning. Moreover, a duplicative attitude causes deficiency in sequence imagination that in particular form is a group of synchronous stimuli ultimately lead to alters behavior ${ }^{17}$. Despite the fact, an individual must be able to integrate sensory inputs for processing either filtering or screening out or suppressing others in functional or operational way. It was observed that most of the brain areas are engage in procession, modulating, and integrating sensory information while the main parts of brain i.e. frontal lobe, cerebellum, superior colliculus having particular focus on quick understanding and rapid multidimensional flow of information, intermediated 
by attentional demands and resources ${ }^{18}$. However, as autism is a mental disorder, the brain processing activity alters in response to the condition. Moreover, this multidimensional sequence of information is impaired in individual of ASD, perhaps this disturbance in the communication of cortical the individual is unable to communicate with their surroundings thereby unable to participate and express their feelings ${ }^{19}$. Though, there would be more interest beyond the influence of attention with autism sensory disorder as an autistic individual faces difficulties with sensory and cognitive domains ${ }^{20}$. Furthermore, people with autism experience more difficulties in automatic processing of knowledge and information that may leads to over loaded attention and stressed working memory therefore when the stimuli reaches and exceeded beyond its capacity, strength and power, thus the processing system fails ${ }^{21}$. Therefore, the purpose of this study is to analyze the sensory processing abilities among autism children. Further the study will be helpful to identify the difficulties faced by autistic children in multidimensional aspects.

\section{METHODOLOGY}

\section{Study Setting}

The study was conducted at special educational schools and institutes of Karachi, Pakistan.

\section{Target Population}

Children diagnosed with Autism Spectrum Children (ASD) aged between 8-12 years.

\section{Study Design \\ Cross-sectional Study.}

\section{Duration of Study}

The study was conducted in the period of 6 months duration.

\section{Sampling Technique \\ Non-probability Convenient Sampling Technique.}

\section{Sample Size}

A Sample Size of 50 was calculated using open EPI calculator.

\section{Sample Selection \\ Inclusion Criteria}

- Children diagnosed with Autism Spectrum Disorders aged 8-12 years

- The children shall be enrolled at Special education school/ institute.

\section{Exclusion Criteria}

- Children with Autism Spectrum Disorders having motor disabilities were excluded.

- Children with associated problems like cerebral palsy, down syndrome

\section{Data Collection Procedure}

Data of 50 participant of age between 8-12 years was collected from special education schools after initial screening based on the given inclusion/ exclusion criteria. Participants were known case of Autism Spectrum Disorder. Consent was taken from their parents/guardians. Procedure of research was explained to them along with the advantages and disadvantages. Short sensory profile was used by the therapist to take information regarding sensory abilities of participants whereas the classification of difference in sensory patterns was analyzed on the total scoring of the profile.

\section{Data Collection Tool}

\section{- Short Sensory Profile}

Data was collected by short sensory profile, consisting of 7 items on tactile sensitivity, taste/smell sensitivity, movement sensitivity, under-responsive/seeks sensation, auditory filtering, low energy/weak, and visual/auditory sensitivity. Each of the items is based on total raw score on the responses of 5-point likert scale i.e. "always", "frequently", "occasionally", "seldom" and "never". The total scoring of each individual was classified on typical, probable and definite difference respectively based on the analysis of sensory processing abilities. The reliability of short sensory profile is $0.70-0.90$ whereas the construct validity of the SSP total and section scores ranges from $(0.25-0.76)^{22-23}$.

\section{- Data Analysis Strategy}

Data was entered and analyzed on IBM SPSS (Statistical Package for Social Sciences) version 20. Descriptive statistics was used to represent the participants' demographic characteristics in terms of frequency, whereas; participants' responses were presented through frequency and percentages chart.

\section{- Ethical Considerations}

Consent from parents/ Guardians was taken before enrolling the participants in the study. They were also informed about the details, possible risks, benefits and their right to withdraw. A secluded area was provided to the participants. Assurance of confidentiality of data was given. Permission from head of the department was also taken for data collection.

\section{RESULTS}

A total of 50 children were recruited after initial screening based on inclusion/exclusion criteria including 23 males and 27 females with mean age of 9.5 \pm 1.84 years. The demographic characteristics of participants are represented in Figure-1.

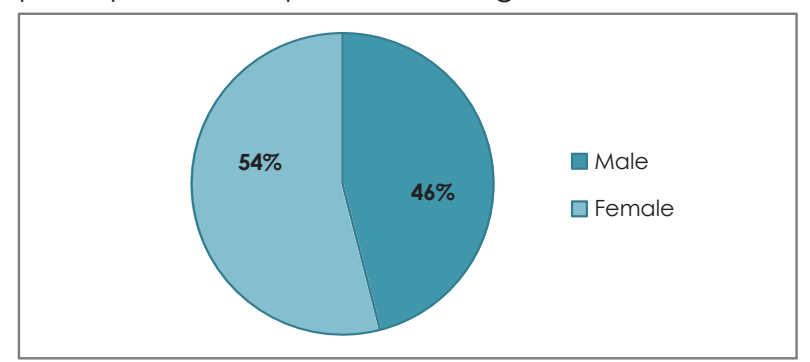

Figure.1 Percentage of Male and Female Participants are represented in pie chart 


\section{Scores on SSP}

On each item of SSP, the mean scores and standard deviation was calculated as shown in Table-2.

\begin{tabular}{|l|l|}
\hline \multicolumn{2}{|l|}{$\begin{array}{l}\text { Table.2 Mean Scores of Participants on } \\
\text { Short Sensory Profile Items }\end{array}$} \\
\hline Items & Mean \pm SD. \\
\hline Tactile Sensitivity & $17.89 \pm 7.61$ \\
\hline Taste/Smell Sensitivity & $11.01 \pm 5.93$ \\
\hline Movement Sensitivity & $9.32 \pm 3.88$ \\
\hline $\begin{array}{l}\text { Under -responsive/Seeks } \\
\text { Sensation }\end{array}$ & $16.66 \pm 6.90$ \\
\hline Auditory Filtering & $17.77 \pm 7.04$ \\
\hline Low Energy/Weak & $19.57 \pm 7.91$ \\
\hline Visual/Auditory Sensitivity & $14.45 \pm 5.75$ \\
\hline
\end{tabular}

Upon analysis, it was observed that no participants were evaluated with probable and typical difference whereas, definite difference was found to be more common. The reported frequency of definite difference is as shown below in Figure 2.

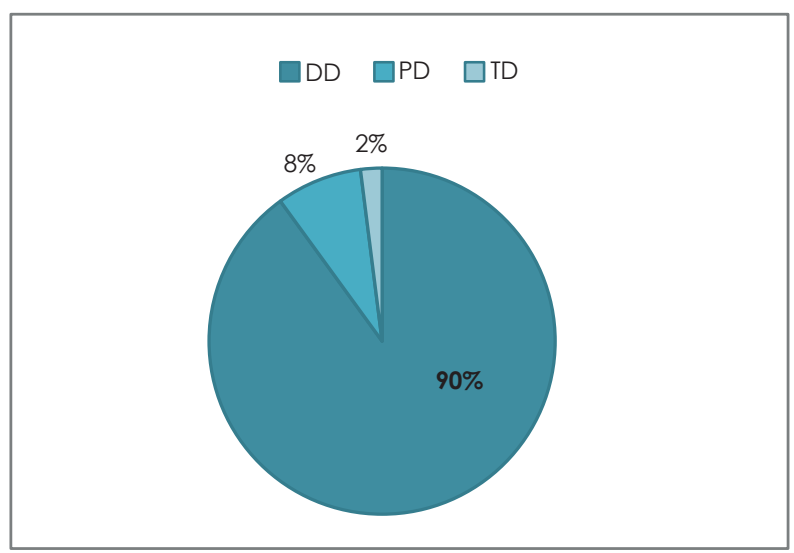

${ }^{*} D D$ : Definite Difference, PD: Probable Difference, TD: Typical Difference

Figure.2 Percentage of participants having different patterns on SSP

\section{DISCUSSION}

The results of this study identified that the children with Autism Spectrum Disorder showed varied responses in sensory integration on Short Sensory Profile. Moreover, definite difference in sensory patterns was observed than probable and typical difference in autism children. These results were consistent with the findings of a study that showed higher prevalence of sensory processing issues in ASD children as compared with normal children ${ }^{24}$. Our study examine the different sensory integration issues with ASD children on Short Sensory Profile on various domains, it was observed that majority of children have low energy, further high scores were found to be in tactile sensitivity, visual and auditory sensitivity that showed the need to address the sensory component in autism children.
Previous studies showed that Autism Spectrum Disorder is associated with more sensory issues as well as poor adaptive functioning that showed the compromised socialization of ASD in activities of daily living and with other people ${ }^{25}$. It was also evident that most of the children with this disorder have atypical presentation on under responsive sensation-seeking item on SSP that revealed that children had no idea regarding their surroundings and they might be lacking in generating the sensory input ${ }^{26-27}$. However, limited studies are found to assess the sensory processing abilities of ASD children with respect to reliability and validity of each item.

Autistic individuals showed varied sensory experiences that include weak or strong responses to certain situations, events or stimulations. Although, the uncomfortable sensory experiences are due to sensory hypersensitivity leads to high or low neurological thresholds causes difficulty in sights, balance, touch, tastes, sound and proprioception. The high neurological threshold causes exaggeration that is worse response in reality whereas low neurological threshold leads to unresponsiveness. Therefore, adequate sensory profile analysis provides possible relationship between behavioral outputs and sensory process abilities to analyze sensory inputs by visual, auditory, olfactory nerves as well by Golgi tendon, tactile receptors and muscle spindle fibers and make connections with cerebral efferent pathway including psychological and emotional junctions ${ }^{28}$.

Furthermore, Lord and Colleagues revealed presence of X-syndrome that is associated with tactile, auditory, taste and smell sensitivity in comparison to other developmental disabilities ${ }^{29}$. Whereas, evidence has been established that stated that sensory symptoms are highest in children with autism. However, limited evaluation measures are available to for comprehensive sensory analysis. Moreover, one of the mechanisms of sensory dysfunction is defensiveness in such a way that they neglect sensory signals to an excessive degree or may refuse it unconsciously that leads to unsupported bodily function and coordination, often observed as difficulties in performing activities in sequential way ${ }^{30}$.

Another study revealed that sub-scales of SSP are strongly associated with autism and sensory processing difficulties; it was also observed that social interaction is the main problem in autistic children are compared to other domains ${ }^{31}$ whereas; our study did not assess the social domain in ASD. The previous studies have reported negative associations between under responsiveness, language and socio communicative symptoms severity score ${ }^{32}$. However, our study did not address the association but majorly highlighted the sensory issues on Short Sensory Profile. On the other hand, Sensory-Seeking patterns have also been related to a higher prevalence of repetitive behaviors $^{33}$. Sensory processing disorder is the condition which affects the ability of brain and nervous system 
to integrate the stimulus, either from the environment or an individual. Therefore, typical responses are observed in affected population. Likewise, children with Autism Spectrum Disorder have altered sensory processing abilities that may affect their senses such as hearing, taste and integration. Therefore, it has been concluded that both under responsive and sensory seeking patterns of interest can have an adverse impact on the performance of individual. The sensory output or responses are interpreted based on child behavioral response to the sensory input from the environment. Our study assessed the sensory measures used in clinical settings but as per the author more interpretation of the behavior pattern and difficulties by the parent or therapist needed to investigate $^{34}$. The study is significant by providing comprehensive assessment of sensory processing abilities of autism spectrum children that is helpful in their rehabilitation. Further, the results difference will provide therapists an area to work in order to improve sensory integration in children with ASD.

The limitations of this study were small sample size and limited age ranges, therefore age-related confounders may vary the sensory profiles. Furthermore, trials must be conducted to investigate the sensory profiles among children with developmental disabilities for more useful outcomes.

\section{CONCLUSION}

It was concluded that definite difference is more prevalent among children with autism whereas varying percentages are found on different items on Short Sensory Profile. Further studies are required to assess the sensory processing issues for more useful outcomes.

\section{REFERENCE}

[1] Townsend RC, Cushion CJ, Smith B. A social relational analysis of an impairment-specific mode of disability coach education. Qualitative Research in Sport, Exercise and Health. $2018 ; 10(3): 346-61$.

[2] Baio J, Wiggins L, Christensen DL, Maenner MJ, Daniels J, Warren Z, Kurzius-Spencer M, Zahorodny W, Rosenberg CR, White T, Durkin MS. Prevalence of autism spectrum disorder among children aged 8 years-autism and developmental disabilities monitoring network, 11 sites, United States, 2014. MMWR Surveillance Summaries. $2018 ; 67(6): 1$.

Christensen DL, Braun KV, Baio J, Bilder D,

[3] Charles J, Constantino JN, Daniels J, Durkin MS, Fitzgerald RT, Kurzius-Spencer $M$, Lee LC. Prevalence and characteristics of autism spectrum disorder among children aged 8 years-autism and developmental disabilities monitoring network, 11 sites, United States, 2012. MMWR Surveillance Summaries. 2018 ;65(13):1.

[4] Seligman M, Darling RB. Ordinary families, special children: A systems approach to childhood disability. Guilford Publications; 2017.

[5] Kuczynski A, Udwin O. Behavioural phenotypes in genetic syndromes associated with intellectual disability. The handbook of intellectual disability and clinical psychology practice. $2016: 431-66$.

[6] Brandes-Aitken A, Anguera JA, Rolle CE, Desai SS, Demopoulos C, Skinner SN, Gazzaley A, Marco EJ. Characterizing cognitive and visuomotor control in children with sensory processing dysfunction and autism spectrum disorders. Neuropsychology. $2018 ; 32(2): 148$.

[7] Singletary WM. An integrative model of autism spectrum disorder: ASD as a neurobiological disorder of experienced environmental deprivation, early life stress and allostatic overload. Neuropsychoanalysis. 2015 ;17(2):81-119.

[8] Brett-Green BA, Rush SF, Shepherd J, Sharpless N, Gavin WJ, Davies PL. A Preliminary Comparison of Multisensory Integration in Boys with Autism Spectrum Disorder and Typically Developing Controls. Int. J. Neurol. 2016;2(1):241-55.

[9] Dakopolos AJ, Jahromi LB. Differences in sensory responses among children with autism spectrum disorder and typical development: Links to joint attention and social competence. Infant and Child Development. $2019 ; 28(1): e 2117$

[10] Pearson N, Charman T, Happé F, Bolton PF, McEwen FS. Regression in autism spectrum disorder: Reconciling findings from retrospective and prospective research.

[11] Autism Research. 2018;11 (12):1602-20.

Gee B, Thompson K, Pierce A, Toupin M, Holst J. The effectiveness of auditory stimulation in children with autism spectrum disorders: A case-control study. Int J Ther Rehabil 2015 ;22(1):36-46.

[12] Gori S, Molteni M, Facoetti A. Visual illusions: An interesting tool to investigate developmental dyslexia and autism spectrum disorder. Frontiers in human neuroscience. 2016 25;10:175.

[13] Bezrukikh MM, Loginova ES, Partsalis EM. Children with impaired cognitive development: Complex assessment and intervention. Human Physiology. 2015;41 (4):356-66.

[14] Torres EB. Commentary on: An exploration of sensory and movement differences from the perspective of individuals with autism. Frontiers in integrative neuroscience. 2015:9.

[15] Glod M, Riby DM, Honey E, Rodgers J. Psychological correlates of sensory processing patterns in individuals with autism spectrum disorder: A systematic review. Review J Autism Dev Disord. 2015 2(2):199-221.

[16] Kendorski JG, Fisher AG. Childhood Autism Spectrum Disorder: Evidence-based Assessment and Intervention. Momentum Press; 2017. 
[17] Shaw SR, Jankowska AM. Pediatric Intellectual Disabilities at School: Translating Research Into Practice. Springer; 2018.

[18] Sokhadze EM, Sears L, El-Baz AS, Tasman A, Casanova MF. Clinical Applications of Electrophysiological Approaches Based on Cortical Modularity in Autism. In Recent Advances on the Modular Organization of the Cortex 2015 (pp. 239-269). Springer, Dordrecht.

[19] Lamm C, Bukowski H, Silani G. From shared to distinct self-other representations in empathy: evidence from neurotypical function and socio-cognitive disorders. Philosophical Transactions of the Royal Society B: Biological Sciences. $2016 ; 371(1686): 20150083$.

[20] South M, Rodgers J. Sensory, emotional and cognitive contributions to anxiety in autism spectrum disorders. Frontiers in Human Neuroscience. 2017 11:20.

[21] Fletcher-Watson S, Happé F. Autism: a new introduction to psychological theory and current debate. Routledge; 2019 .

[22] Davis AM, Bruce AS, Khasawneh R, Schulz T, Fox C, Dunn W. Sensory processing issues in young children presenting to an outpatient feeding clinic: A retrospective chart review. Journal of pediatric gastroenterology and nutrition. 2013 ;56(2):156.

[23] Lane AE, Molloy CA, Bishop SL. Classification of Children With A utism S pectrum D isorder by Sensory Subtype: A Case for Sensory-Based Phenotypes. Autism Research. 2014;7(3):322-33.

[24] Hazen EP, Stornelli JL, O'Rourke JA, Koesterer K, McDougle CJ. Sensory symptoms in autism spectrum disorders. Harvard review of psychiatry. 2014;22(2):112-24.

[25] Crasta J, Davies PL. Behavioral and Neural Sensory Processing in Young Adults With Autism Spectrum Disorder. Am J Occup Ther. 2018 ;72(7211505081pl-.

[26] Dellapiazza F, Michelon C, Oreve MJ, Robel L, Schoenberger M, Chatel C, Vesperini S, Maffre T, Schmidt R, Blanc N, Vernhet C. The Impact of Atypical Sensory Processing on Adaptive
Functioning and Maladaptive Behaviors in Autism Spectrum Disorder During Childhood: Results From the ELENA Cohort. J Autism Dev Disord2019 13:1-1.

[27] Green SA, Hernandez L, Tottenham N, Krasileva K, Bookheimer SY, Dapretto $M$. Neurobiology of sensory overresponsivity in youth with autism spectrum disorders. JAMA psychiatry. $2015 ; 72(8): 778-86$.

[28] Martin ST, Kessler M. Neurologic interventions for physical therapy. Elsevier Health Sciences; 2015 .

[29] Kolacz J, Raspa M, Heilman KJ, Porges SW. Evaluating sensory processing in fragile $X$ syndrome: Psychometric analysis of the brain body center sensory scales (BBCSS). J Autism Dev Disord. $2018 ; 48(6): 2187-202$.

[30] Matthews M, Bell E, Mirfin-Veitch B. Comparing psychopathology rates across autism spectrum disorders and intellectual disabilities. Advances in Mental Health and Intellectual Disabilities. 2018

[31] Sperdin HF, Coito A, Kojovic N, Rihs TA, Jan RK, Franchini M, Plomp G, Vulliemoz S, Eliez S, Michel CM, Schaer M. Early alterations of social brain networks in young children with autism. ELife. $2018 ; 7:$ e31670.

[32] Zarafshan $H$, Salmanian $M$, Aghamohammadi S, Mohammadi MR, Mostafavi SA. Effectiveness of non-pharmacological interventions on stereotyped and repetitive behaviors of pre-school children with autism: a systematic review. Basic and clinical neuroscience. 2017;8(2):95.

[33] Zheng L, Grove R, Eapen V. Spectrum or subtypes? A latent profile analysis of restricted and repetitive behaviours in autism. Research in Autism Spectrum Disorders. $2019 ; 57: 46-54$.

[34] Ausderau KK, Furlong $M$, Sideris J, Bulluck J, Little LM, Watson LR, Boyd BA, Belger A, Dickie VA, Baranek GT. Sensory subtypes in children with autism spectrum disorder: latent profile transition analysis using a national survey of sensory features. Journal of Child Psychology and Psychiatry. 2014 55(8):935-44. 